NASA Technical Memorandum $83282 \quad$ NASA-TM-83282 19820016982

\title{
RECENT DEVELOPMENTS IN TRANSONIC EULER FLOW OVER A CIRCULAR CYLINDER
}

Manuel D. Salas

APRIL 1982

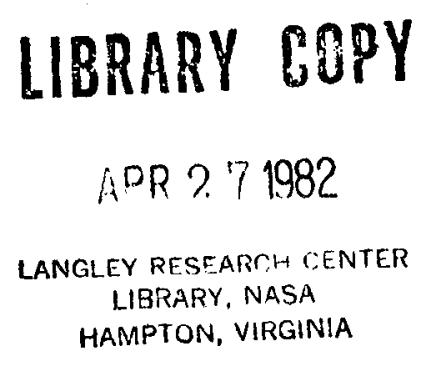

N/Sก

National Aeronautics and Space Administration

Langley Research Center Hampton, Virginia 23665 



\section{SUMMARY}

Numerical solutions to the Euler equations for transonic flow over a circular cylinder indicate that the inviscid flow separates ahead of the rear stagnation point. Our understanding of this phenomenon and various solutions presented at a workshop on this subject are discussed.

\section{INTRODUCTION}

At high speeds, as air rushes past a circular cylinder, a pocket of supersonic flow terminated by a recompression shock forms near the top of the cylinder. Recent numerical calculations made by the author, based on the inviscid Euler equations, also showed a bubble of recirculating flow at the rear of the cylinder. Since separation is usually associated with the vorticity generated at solid boundaries by viscosity, its occurrence in these inviscid calculations was at first thought to be an artifact of the numerical simulation. However, after many careful calculations to determine the effects of the initial conditions, the boundary conditions, and the artificial viscosity inherent in the numerical scheme, the phenomenon appeared to be real (i.e., consistent with the Euler equations). Further support for the validity of the recirculating bubble as a solution of the Euler equations came from the theoretical studies in references 1 and 2 , where it was shown that inviscid separation can occur in rotational flows as a result of the premature retardation of the surface velocity caused by vorticity in the flow. The vorticity in this case being generated by the recompression shock. In order to gain further insight, and to have these results independentIy verified, a workshop was held at NASA Langley 
Research Center on September 1, 1981. The main purpose of this paper is to review the results presented at the workshop.

\section{RESULTS PRESEN'IED AT THE WORKSHOP}

Of the eight presentations given at the workshop, two were in a preliminary state of development and will not be discussed here. The remaining six talks described the work of Mohamed Hafez of George Washington University; Ron-Ho Ni of Pratt and Whitney; Joseph Steger of Stanford University; Eli Turkel of Tel Aviv University, Israel; Bram van Leer of Leiden State University, The Netherlands; and the author (refs. 3-8). (References cited give details of the numerical schemes used, but they do not address the problem discussed here.) The talks concentrated on three cases corresponding to free-stream Mach numbers $\left(M_{\infty}\right)$ of $0.4,0.5$, and 0.6 . The $M_{\infty}=0.4$ case corresponds to the incipient formation of the supersonic bubble. This case offers a good check on the results since the flow is still potential and should have front and aft symmetry. All results presented for this case showed good qualitative and quantitative agreement. However, a slight front/aft asymmetry could be observed in the results of $\mathrm{Ni}$, Turkel, and van Leer. Figure 1 shows the streamline pattern for this case as computed by the author and figure 2 the Mach number contours from van Leer's calculation.

At $M_{\infty}=0.5$, all calculations, except for van Leer's which was much smaller, found a recirculating bubble of approximately a diameter in width, see figures 3 and 4 , and were in qualitative agreement. Some quantitative differences were, however, observed at this Mach number among the various calculations, particularly in the details of the recirculating bubble. For example, in figure 5 the computation by $\mathrm{Ni}$ 
shows the pressure within the recirculating bubble to be essentially constant; while the author finds a substantial variation in the pressure in this region.

At the higher free-stream Mach number, $M_{\infty}=0.6$, the calculation of Turke1, Steger, van Leer, and the author shows a very long recirculating bubble, possibly extending to infinity; see figure 6 . At this Mach number the calculations of both $\mathrm{Ni}$ and Hafez failed to converge. The technique used by Hafez is interesting in that it points to the possible existence of multiple solutions satisfying the steadystate Euler equations. Hafez solves the steady-state equations by introducing a stream function. The resulting second-order partial differential equation is very similar to the full-potential equation but with the vorticity acting as a source term, and it is thus solved by standard relaxation methods. Since the vorticity divided by the pressure is only a function of the stream function, the source term is easily evaluated at any point downstream of the shock wave by tracing the streamline back to the shock wave. However, for a point within the recirculating bubble, the streamlines form a closed path and their level of vorticity cannot be evaluated by tracing them back to the shock. Hafez believes that this indicates the existence of multiple solutions depending on how the level of vorticity is modeled within the bubble. In his computations, he uses extrapolation from the region outside the bubble to obtain the vorticity levels inside the bubble. It should be pointed out that the other methods reported here alI solve the time-dependent Euler equations which allow for the formation of the bubble in time without need for an explicit model of the vorticity within. 
The drag coefficient was available from four of the calculations reported here. In Table 1 they are compared to the drag coefficient from a conservative potential calculation. Although there is a considerable spread in the values for the Euler calculations, they all indicate a lower drag than predicted by the potential calculation. This comes about because in the Euler calculation the shock wave occurs ahead of the potential shock and is therefore weaker, and also because the recirculating bubble occurring in the Euler calculations, unlike the separation bubble in a typical viscous flow, is a region of high near-stagnation pressure which produces thrust rather than drag.

An interesting discovery was made by Steger while performing his calculations. Rather than limiting his computations to the upper half plane and imposing flow symmetry along the center line, as the other investigators did, Steger computed the full $360^{\circ}$ circle. For these calculations, Steger noticed that the flow at the rear of the cylinder oscillated, preventing convergence to a steady state. This phenomenon has since been reproduced by Turkel and the athor. In the calculations of steger it is triggered by an inherent asymmetry in the approximate factorization technique used; while in the calculations by Turkel and the author, it is necessary to introduce an asymmetry into the initial ccnditions in order to observe it. The oscillations are only observed for supercritical cases and seem to be sustained by reflection of waves from the upper and lower shock waves. 


\section{CONCLUDING REMARKS}

All the evidence now available indicates that inviscid separation is a feature of the Euler equations induced by vorticity and/or stagnation pressure loss in the flow. The possibility of multiple solutions suggested by Hafez and the oscillatory behavior for the full circle observed by Steger need further investigation.

Further studies of this problem may shed some light in determining the relevant Euler solution of the Navier-Stokes equation in the limit of vanishing viscosity for separated flows, a problem which Saffman (ref. 9) considers as one "of the challenging unsolved problems of fluid mechanics." 


\section{REFERENCES}

1. Fraenke1, Lo E.: On Cornex Iddies in Plane Inviscid Shear Flow. J. Fluid Mech., vol. 11, 1261, pp. 400-406.

2. Küchemann, D.: Inviscid Shear Flow Near the Trailing Edge of an Airfoil. Z. Flugwiss, vol. 15, 1967, pp. 292-294.

3. Hafez, M.; and Lovel1, D.: Numerical Solution of Transonic Stream Function Fquation. AIAA Papex 81-1017, A Collection of Technical Papers, AIAA 5th Computational Fluid Dynamics Conference, 1981, pp. 364-372.

4. Ni, R. H.: A Multiple Grid Scheme for Solving the Euler Equations. AIAA Paper 81-1025, A Collection of Technical Papers, AIAA 5th Computational Fluid Dynamics Conference, 1981, pp. 257-261.

5. Steger, J. L.: Implicit Finite Diffexence Simulation of Flow About Arbitrary Geometrios with Applications to Airfoils. AIAA Paper 77-665, 1977.

6. Jameson, A.; Schmidt, W.; and Turkel, E.: Numerical Solutions of Euler Equations by Finite Volume Methods Using Runge-Kutta TimeStepping Schemes. AIAA Paper 81-1259 presented at AIAA 14th Fluid. and Plasma Dynamics Conference, June 23-25, 1981.

7. van Leer, Bram: Towards the Ultimate Conservative Difference Scheme. V. A second-Order Sequel to Godunov's Method. J. Comp. Phys., vol. 32, no. 1, 1979, pp. 101-136.

8. Moretti, G.: The $\lambda$-Scheme. Comp. and Flds., vo1. 7, 1979, pp. 191-205.

9. Saffman, P. G.: Dynamics of Vorticity, J. Fluid Mech., vol. 106, 1981, pp. 49-58. 
TABLE 1.- DRAG COEFFICIENT COMPUTED BY VARIOUS METHODS

\begin{tabular}{|c|c|c|c|c|c|}
\hline \multirow{2}{*}{$M_{\infty}$} & \multirow{2}{*}{$\begin{array}{c}\text { Conservative } \\
\text { Potential }\end{array}$} & \multicolumn{4}{|c|}{ Euler } \\
\hline & & $\mathrm{Ni}$ & Salas & Steger & Turkel \\
\hline 0.40 & -0.0000 & 0.0124 & -0.0013 & 0.0062 & 0.0033 \\
\hline 0.50 & 0.3799 & 0.1733 & -0.0041 & 0.1794 & 0.0617 \\
\hline 0.60 & 1.1293 & $\begin{array}{c}\text { Not } \\
\text { available }\end{array}$ & 0.0618 & 0.6066 & 0.1628 \\
\hline
\end{tabular}




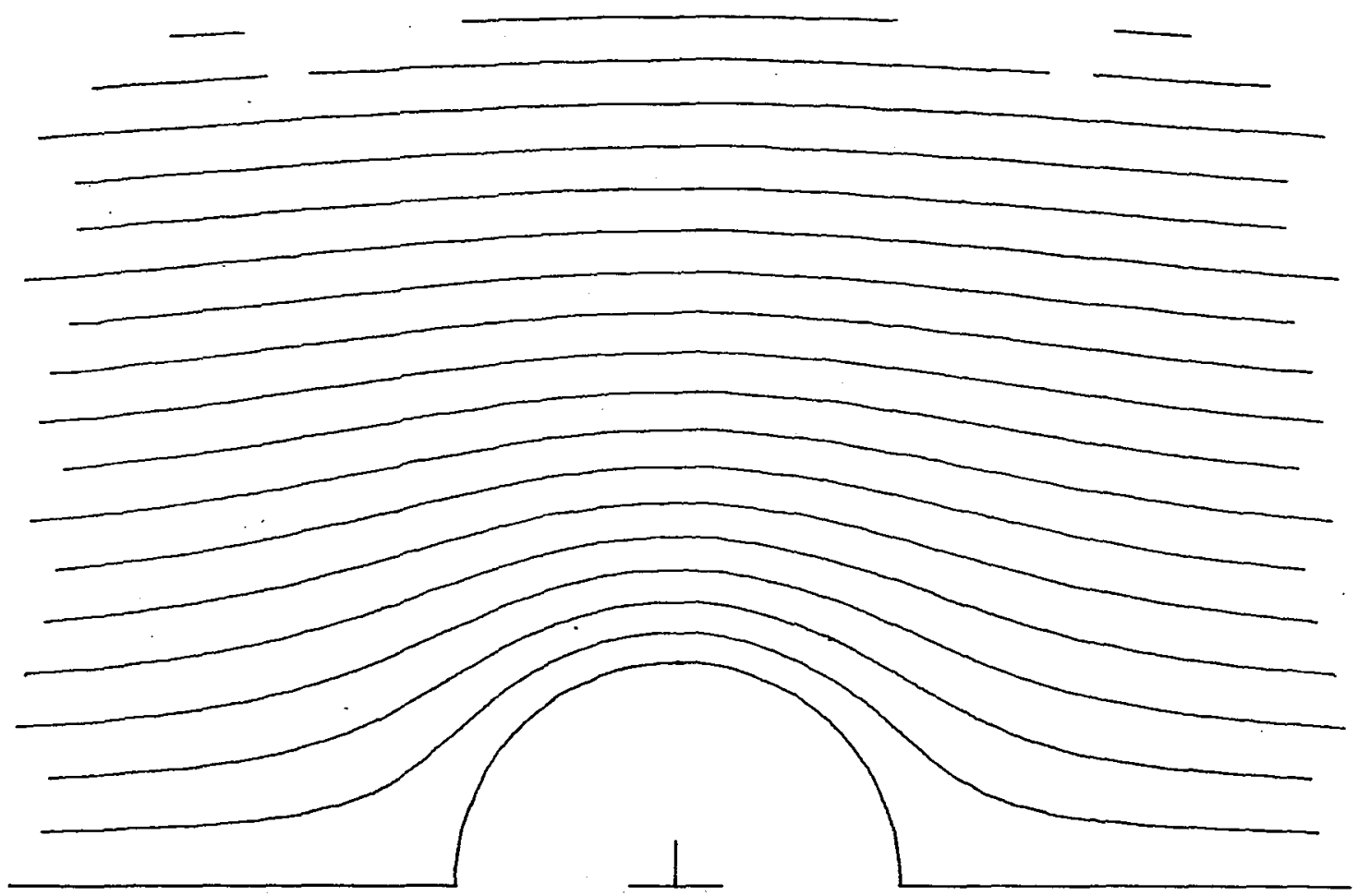

Figure 1.- Streamline pattern for $M_{\infty}=0.4$ calculated by the author. 


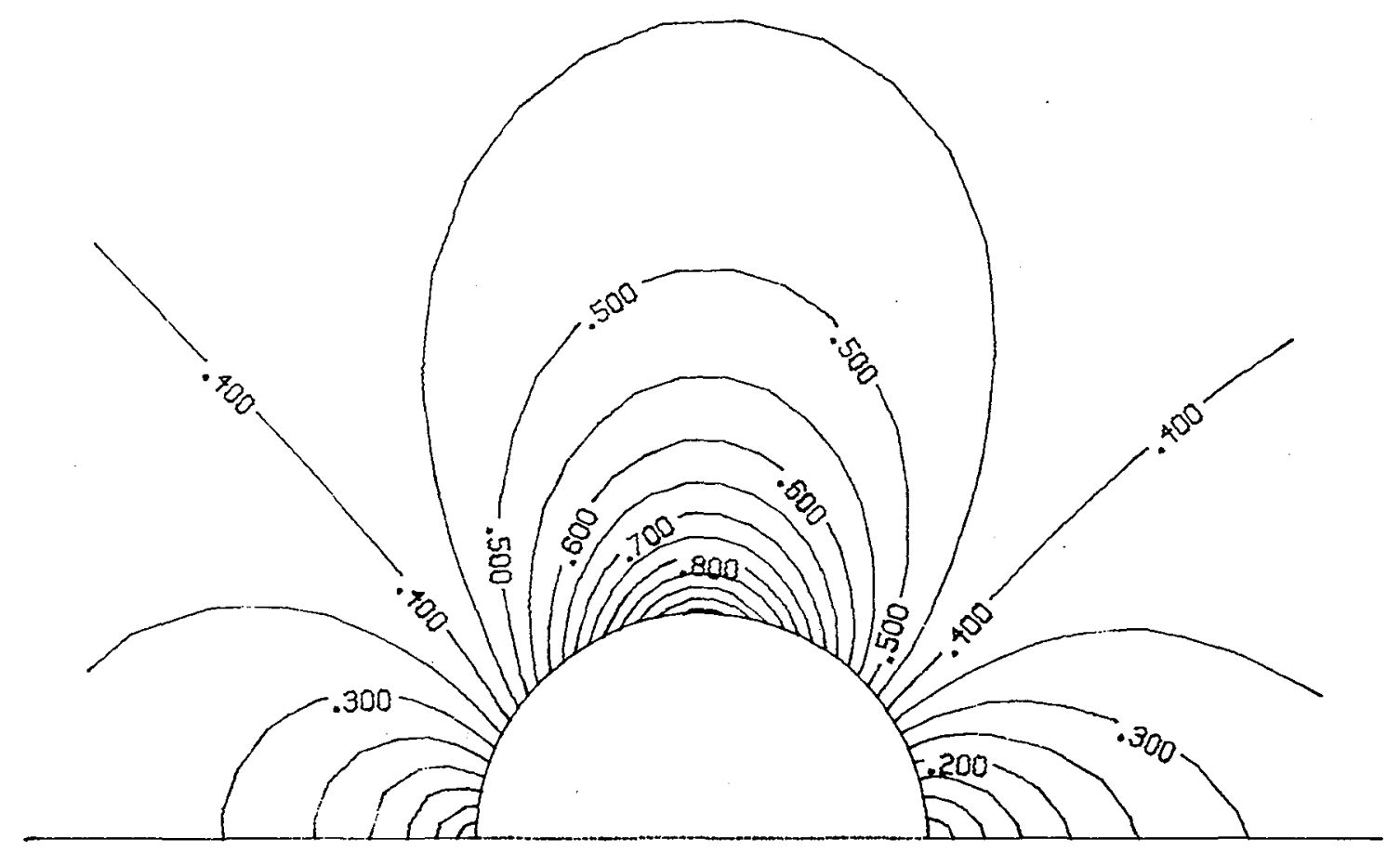

Figure 2.- Mach number contours for $M_{\infty}=0.4$ calculated by van Leer. Notice the slight front/aft asymmetry. 


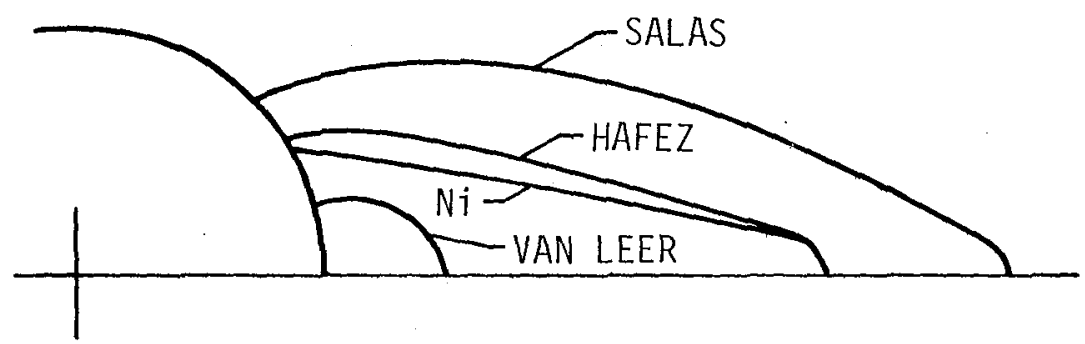

Figure 3. - Comparison of recirculation bubble shape at $M_{\infty}=0.5$. 


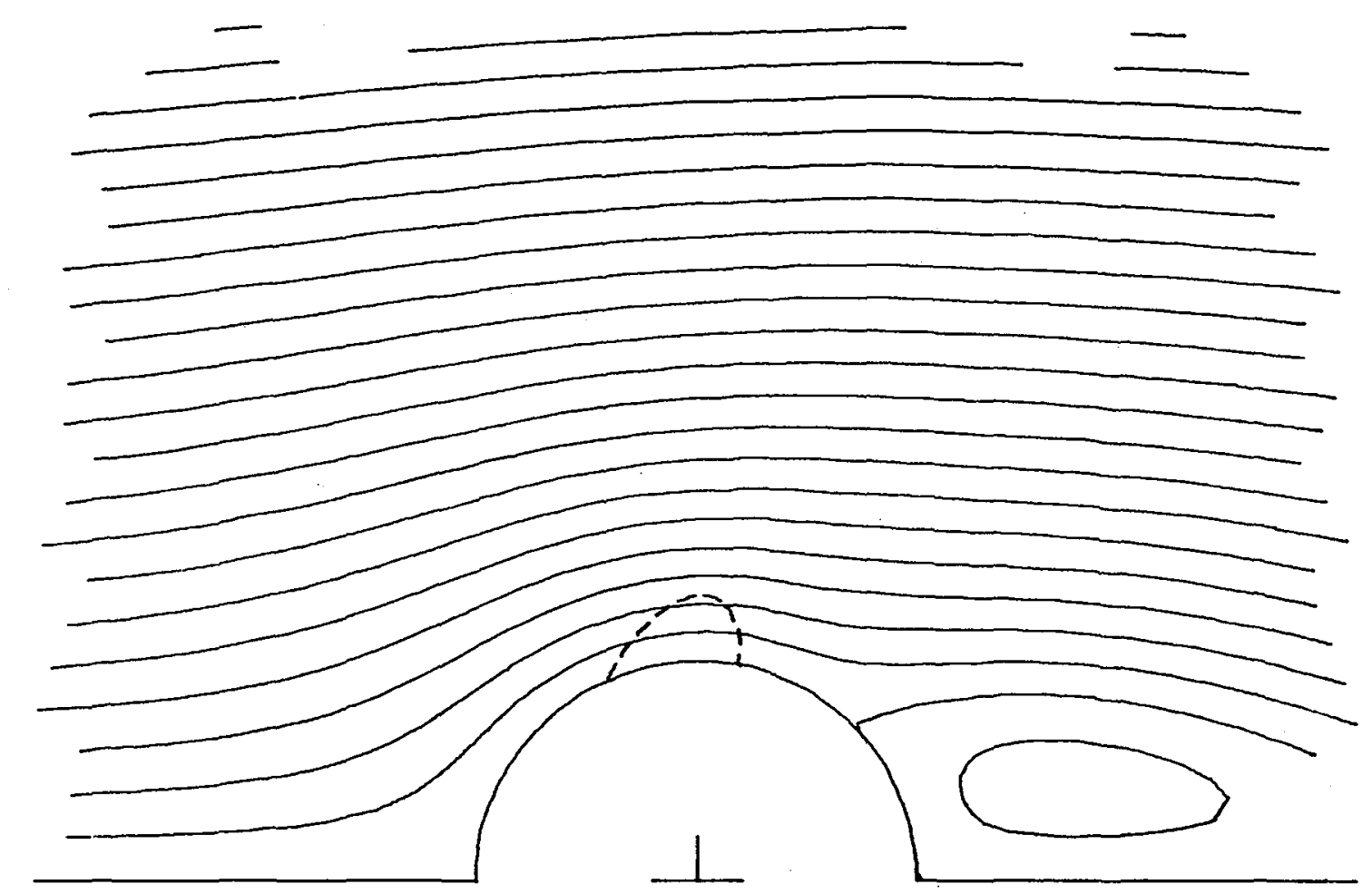

Figure 4.- Streamline pattern for $M_{\infty}=0.50$ calculated by the author. Sonic line shown as a dashed line. 


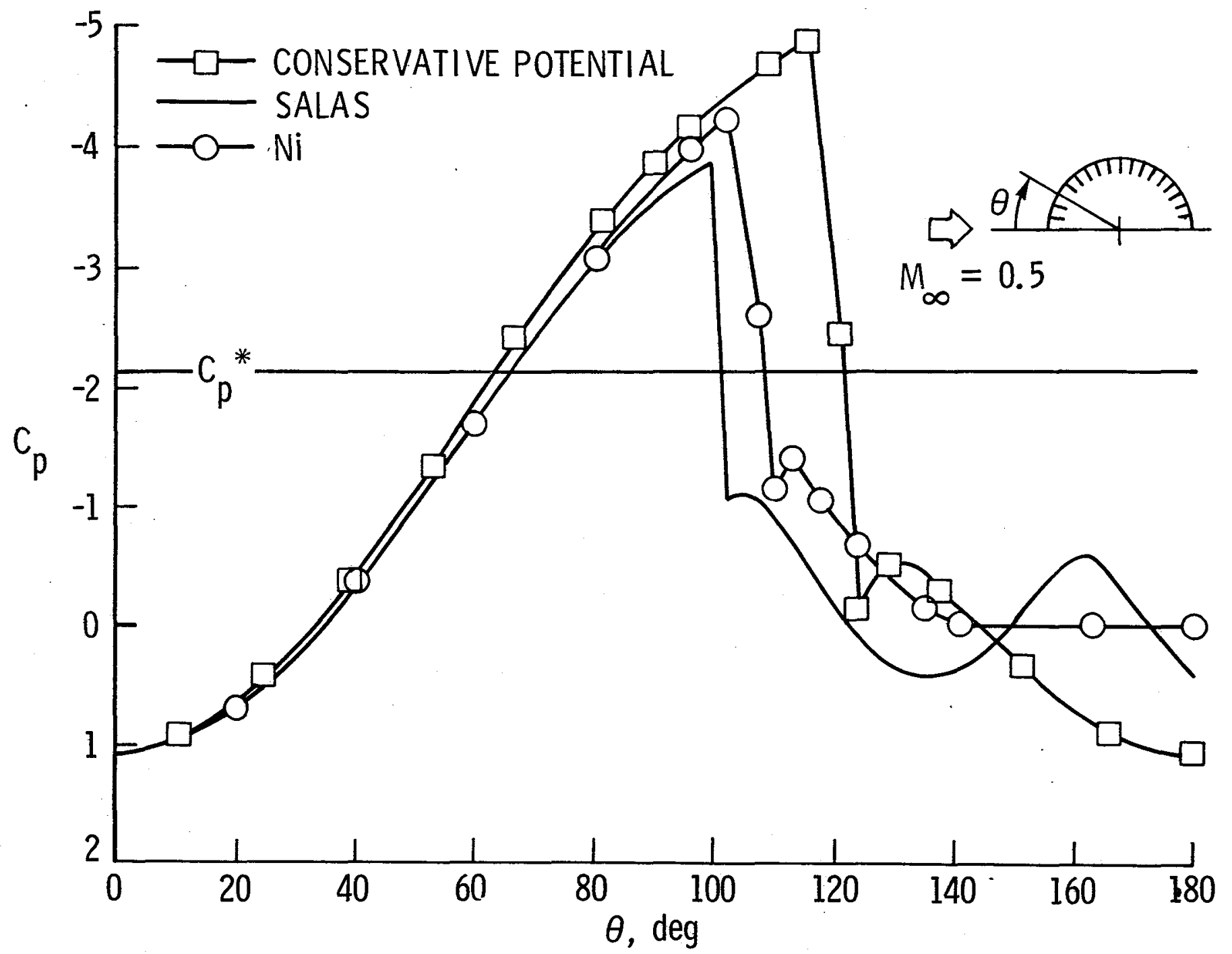

Figure 5.- Pressure coefficient at $M_{\infty}=0.50$ computed by Ni and Salas. Results of a conservative potential calculation are included for comparison. 


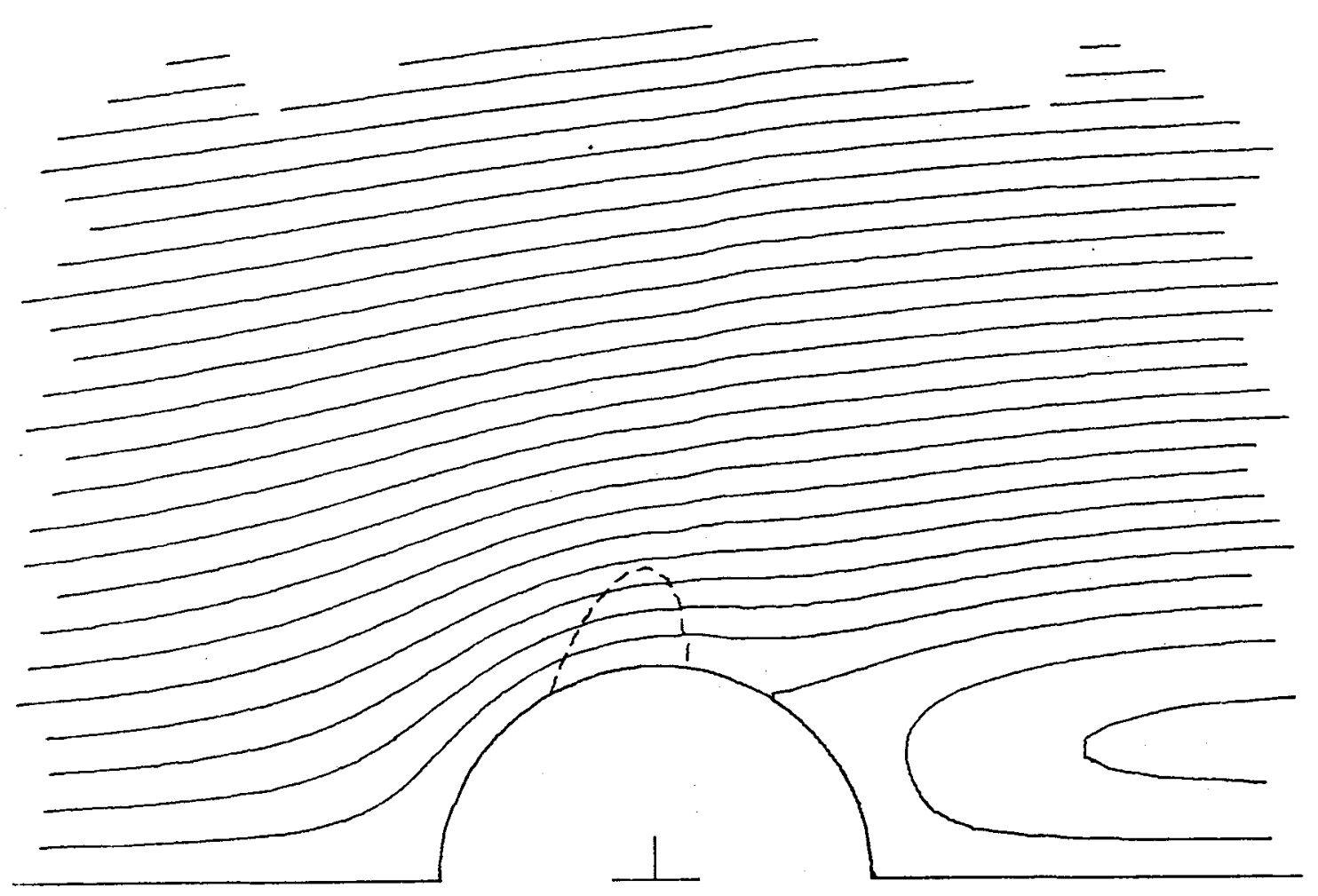

Figure 6.- Streamline pattern for $M_{\infty}=0.6$ calculated by the author. 




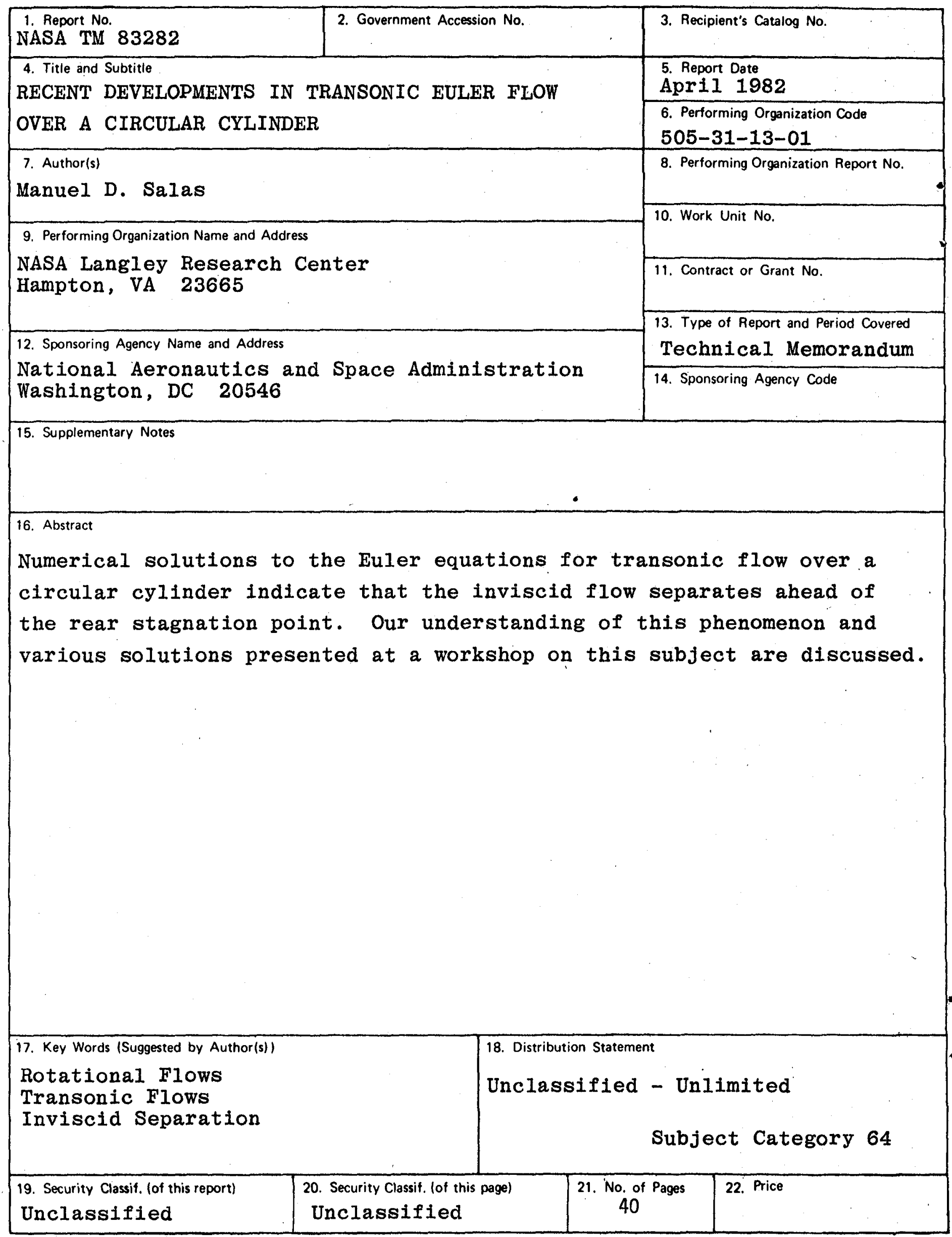



